\title{
Stellar elemental abundances constraining nucleosynthe- sis and chemical evolution of the universe
}

\author{
Wako Aoki ${ }^{1,2, *}$ \\ ${ }^{1}$ National Astronomical Observatory of Japan, 2-21-1, Osawa, Mitaka, Tokyo 181-8588, Japan \\ ${ }^{2}$ Department of Astronomical Science, School of Physical Sciences, The Graduate University of Ad- \\ vanced Studies (SOKENDAI), 2-21-1, Osawa, Mitaka, Tokyo 181-8588, Japan
}

\begin{abstract}
Measurements of elemental abundances of stars in the Milky Way and surrounding dwarf galaxies in the past few decades have been providing useful constraints on modeling the nucleosynthesis and chemical evolution. Recent large spectroscopic surveys combined with studies on dynamical motions of stars in the Milky Way reveal connections between chemical enrichment and galaxy formation. This paper provides a brief overview of the recent update of the understanding of the distribution of elemental abundances and remaining issues for key elements, mostly focusing on the low metallicity range which should reflect nucleosynthesis by massive stars and early chemical evolution. This covers the abundance ratios of $\alpha$-elements, iron-peak elements, and neutron-capture elements which have been measured for a large number of metal-poor stars.
\end{abstract}

\section{Introduction}

The cycle of formation, evolution, and death of stars, including interactions of binary stars, have been enriching heavier elements in the Universe. Low-mass stars formed in the early Universe could survive in the present Universe thanks to their long lifetime, and are observed as metal-poor stars. The elemental abundances of very metal-poor stars should reflect the results of nucleosynthesis by a small number of stars and phenomena in the early Universe. Hence, spectroscopic observations of metal-poor stars provide unique constraint on elemental abundances produced by individual nucleosynthesis events.

Since the evolution timescale of massive stars is short, the elemental abundances of very metal-poor stars are mostly determined by massive star evolution and supernova explosions. Taking account of the dependence of elemental abundance patterns produced by supernovae on their progenitor masses, abundance studies of very metal-poor stars are also able to estimate the masses of early generations of stars $[1,2]$.

Distributions of abundance ratios of individual elements for a large sample of metalpoor stars are useful constraints on chemical evolution models (Kobayashi, this volume). For instance, abundance ratios of $\alpha$ and iron-peak elements (e.g., $[\mathrm{Mg} / \mathrm{Fe}])^{1}$ as a function of metallicity are used as an indicator of contributions of core-collapse supernovae and type

\footnotetext{
*e-mail: aoki.wako@nao.ac.jp

${ }^{1}[\mathrm{~A} / \mathrm{B}]=\log \left(N_{\mathrm{A}} / N_{\mathrm{B}}\right)-\log \left(N_{\mathrm{A}} / N_{\mathrm{B}}\right)_{\odot}$, and $\log \epsilon_{\mathrm{A}}=\log \left(N_{\mathrm{A}} / N_{\mathrm{H}}\right)+12$ for elements $\mathrm{A}$ and $\mathrm{B}$.
} 
Ia supernovae, which reflect the timescale of chemical evolution of the substructures of the Milky Way Galaxy and dwarf galaxies.

Studies of elemental abundances for early generations of stars have been led by large surveys of metal-poor stars since 1980s. The HK survey [3] and the Hamburg/ESO survey [4] have applied objective prisms on Schmidt telescopes to select candidates for metal-poor stars from the absorption lines of ionized $\mathrm{Ca}$ (the so-called $\mathrm{Ca} \mathrm{H}$ and $\mathrm{K}$ lines) and other features. High-resolution follow-up spectroscopy was conducted for the candidates in 1990s (e.g., [5, 6]), and later for larger samples with the 8-10 m-class telescopes [7-9]. Since 2000s, metal-poor stars have been also searched for using multi-object spectrometers, e.g., Sloan Digital Sky survey [10] and LAMOST [11]. Another approach is to conduct photometry using narrow-band filters designed to investigate absorption features that are sensitive to stellar metallicity (e.g., the Ca H-K lines). Skymapper survey [12] and Pristine survey [13] are examples of photomeric surveys that provide large samples of candidate very metal-poor stars.

We here discuss the recent results based on the follow-up high-resolution spectroscopy with the Subaru Telescope for candidates of metal-poor stars found by the LAMOST survey [14]. We note that the abundance measurements for a large sample of metal-poor stars found by the Skymapper survey recently reported similar results for many elements [15] .

\section{Elemental abundances of metal-poor stars}

A large sample ( 10,000 stars) of candidates for very metal-poor stars was obtained from the pilot and regular survey programs with LAMOST (e.g., [16]). High-resolution spectra of about 400 stars were obtained with the Subaru Telescope High Dispersion Spectrograph (HDS) in 2014-2017 [17]. Detailed elemental abundances are determined for these stars [14]. The sample mostly consists of main-sequence turn-off stars and red giants. The remaining stars are horizontal-branch stars that are on helium core burning phase in low-mass star evolution. The evolutionary status is well determined for most of the stars thanks to the accurate distances recently obtained by the trigonometric parallaxes provided by the Gaia mission [18].

The sample covers $[\mathrm{Fe} / \mathrm{H}]<-2$. In this metallicity range, stars in the halo component of the Milky Way is dominant.

Elemental abundances are determined using standard analysis of spectral lines using 1D LTE model atmospheres [19]. The analysis method and their behaviors have been well studied for metal-poor stars, and, hence, the results can be compared with previous studies. In this section, abundance trends are discussed for individual elements compared with chemical evolution models based on the recent results [14]. We here refer to three chemical evolution models [20-22].

\section{$2.1 \alpha$-elements (Mg, Si, Ca, Ti) and Sc}

Abundance ratios between $\alpha$-elements and Fe generally show over-abundances (i.e., $[\alpha / \mathrm{Fe}]>$ $0)$. This is a signature of dominant contributions of core-collapse supernovae in the early phase of chemical evolution, reflecting their short time-scale. Closer inspection of abundance ratios of individual elements provides useful constraints on, or suggestion for, the chemical evolution and the structure formation of the Milky Way halo.

The $[\mathrm{Mg} / \mathrm{Fe}]$ and $[\mathrm{Ca} / \mathrm{Fe}]$ values exhibit decreasing trends with increasing metallicity in $[\mathrm{Fe} / \mathrm{H}] \gtrsim-2.5$ (Fig. 1). A fraction of stars are known to have low $[\alpha / \mathrm{Fe}]$ at higher metallicity $([\mathrm{Fe} / \mathrm{H}] \gtrsim-1.5)[23]$, and are interpreted as a result of accretion of stellar systems similar to 

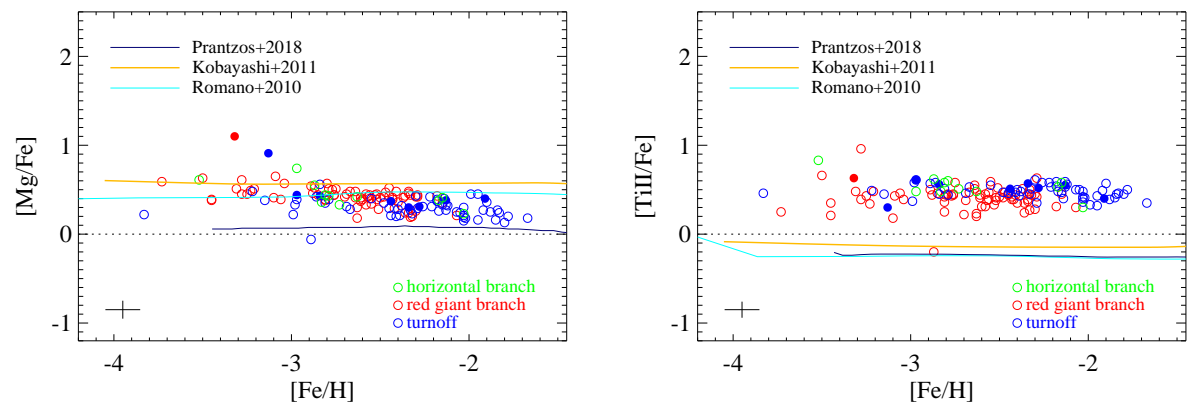

Figure 1. Abundance ratios $([\mathrm{Mg} / \mathrm{Fe}]$ and $[\mathrm{Ti} / \mathrm{Fe}])$ as a function of $[\mathrm{Fe} / \mathrm{H}]$. Red and blue circles indicate the abundance results for red giants and main-sequence turn-off stars, respectively [14]. Predictions of the three chemical evolution models [20-22] are shown by lines as presented in the panels.

current dwarf galaxies, in which the timescale of the chemical evolution is longer [24]. Such effects might already appear at lower metallicity. Si might show a similar trend, but it is not as clear as the above two elements due to the limited size of the sample and data quality. The situation will be improved by observations with new near-infrared spectrometers that covers the wavelength range in which many Si lines useful for accurate abundance measurements exist [25].

Ti also shows over-abundance as found for $\mathrm{Mg}, \mathrm{Si}$ and $\mathrm{Ca}$ (Fig. 1). This result is obtained from analyses of neutral and ionized Ti lines, indicating that the result is robust. The [Ti/Fe] values are almost constant in this metallicity range. This overabundance is not explained by any of the three chemical evolution models. The discrepancy would be due to the limited understanding of the yields of core-collapse supernovae.

Sc, an element with odd atomic number, also exhibits over-abundances, which are not reproduced by chemical evolution models as in the case of Ti.

Abundance ratios of $\alpha$-elements, with respect to $\mathrm{Fe}$, show correlations each other. The correlations are particularly strong between $[\mathrm{Mg} / \mathrm{Fe}]$ and $[\mathrm{Si} / \mathrm{Fe}]$, and also between $[\mathrm{Ca} / \mathrm{Fe}]$ and $[\mathrm{Ti} / \mathrm{Fe}]$. The former are lighter $\alpha$-elements, representing the products during massive star evolution, whereas the later heavier ones are products of explosive nucleosynthesis. The correlations between the lighter and heavier $\alpha$-elements are less significant. [Sc/Fe] shows clear correlations with $[\mathrm{Ca} / \mathrm{Fe}]$ and $[\mathrm{Ti} / \mathrm{Fe}]$, suggesting that the elements have similar origins.

\subsection{Iron-peak elements}

The $[\mathrm{Cr} / \mathrm{Fe}]$ values determined for red giants show a clear decreasing trend with decreasing metallicity, whereas those for main-sequence stars are almost constant (Fig. 2). The trend found in red giants is considered to be caused by the non-LTE effect in the line formation of neutral $\mathrm{Cr}$ in very metal-poor stars. Taking account of this effect, $\mathrm{Cr}$ abundance ratios agree well with the solar value, as found for $\mathrm{Ni}$. The $[\mathrm{Mn} / \mathrm{Fe}]$ values are clearly lower than the solar value. These results are well reproduced by the chemical evolution models. On the other hand, Co shows slight over-abundance, which is not explained by models that predict the solar abundance ratio for very metal-poor stars. $[\mathrm{Zn} / \mathrm{Fe}]$ shows increasing trend with decreasing metallicity (Fig. 2). The sample [14] does not well cover the lowest metallicity range $[\mathrm{Fe} / \mathrm{H}]<$ -3.5 due to the limited data quality. This could lead selection bias in the abundance trend as discussed [15]. However, previous studies covering the low metallicity range (e.g., [7]) report that the trend continues down to $[\mathrm{Fe} / \mathrm{H}] \sim-4$. The chemical evolution models with standard 

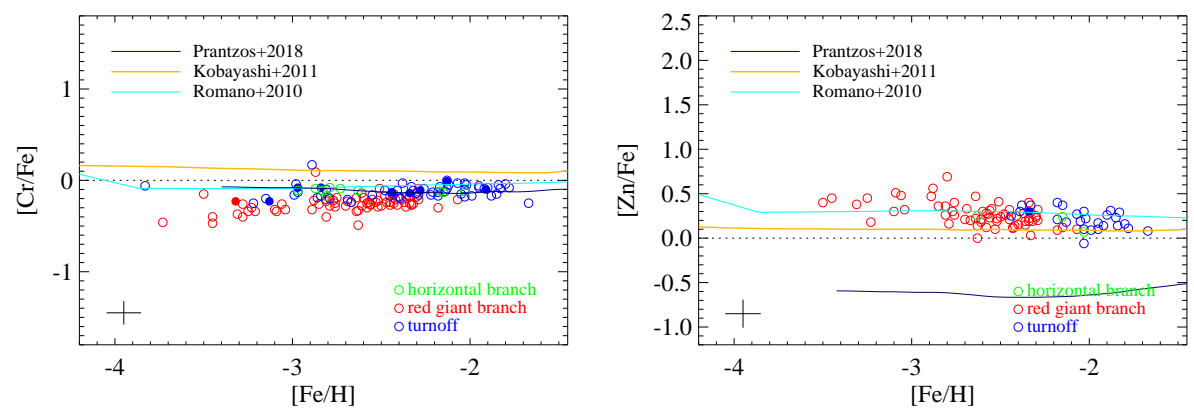

Figure 2. The same as Fig. 1, but for $\mathrm{Cr}$ and $\mathrm{Zn}$.

yields predict lower abundance ratios of $\mathrm{Zn}$. This suggests that contributions of energetic supernova explosions (e.g., hypernovae) are required to be included.

$\mathrm{Zn}$ abundance ratios do not show clear correlations with those of $\alpha$-elements. The exception is the $\mathrm{Ti}$ abundance ratios, that exhibit a significant correlation with the $\mathrm{Zn}$ abundance ratios, suggesting a connection between the production site of these elements.

\subsection{Neutron-capture elements}

The heavy neutron-capture elements $\mathrm{Sr}$ and $\mathrm{Ba}$ are relatively abundant and have strong spectral lines in the optical range and, then, are useful for investigating the distributions of abundance ratios at very low metallicity. A large scatter in both $[\mathrm{Sr} / \mathrm{Fe}]$ and $[\mathrm{Ba} / \mathrm{Fe}]$ is a wellknown property. However, a closer inspection reveals different features between them. In $[\mathrm{Fe} / \mathrm{H}]>-3$, most of the stars have $[\mathrm{Sr} / \mathrm{Fe}]$ and $[\mathrm{Ba} / \mathrm{Fe}]$ close to 0 (i.e., solar abundance ratio), but $\mathrm{Sr}$ shows stronger concentration. The large scatter of $\mathrm{Sr}$ and $\mathrm{Ba}$ abundance ratios $(-2<\lesssim[\mathrm{Sr} / \mathrm{Fe}],[\mathrm{Ba} / \mathrm{Fe}] \lesssim+1)$ is found in $[\mathrm{Fe} / \mathrm{H}] \lesssim-3$. While $[\mathrm{Sr} / \mathrm{Fe}]$ values show a rather flat distribution in the above range, a large fraction of stars have $[\mathrm{Ba} / \mathrm{Fe}] \sim-1$. The $[\mathrm{Sr} / \mathrm{Ba}]$ values also show a wide distribution. This suggests the existence of different origins for $\mathrm{Sr}$ and $\mathrm{Ba}$ at very low metallicity. We note that the so-called carbon-enhanced mtal-poor stars with $\mathrm{Ba}$ excess (CEMP-s) are not included in this discussion.

A small fraction of stars with $[\mathrm{Fe} / \mathrm{H}]<-3$ have very low $\mathrm{Sr}$ and $\mathrm{Ba}$ abundance ratios ([Sr/Fe], $[\mathrm{Ba} / \mathrm{Fe}] \sim-2)$. There is no constraint on their origin from other elements (e.g., $\mathrm{Eu})$. Further studies are required for these stars to understand the possible source of such small amount of neutron-capture elements. The origins of neutron-capture elements are also discussed in the conference (Tarumi et al., this volume).

\section{Summary and prospects}

Measurements of elemental abundances for a large sample of metal-poor stars have been revealing the origins and the evolution of elements in the Universe. The studies also identify problems to be solved in comparisons between observations and models for abundance distributions. For instance, the Ti and Sc over-abundances, and the slight over-abundance of Co and overabundance of $\mathrm{Zn}$ at extremely low metallicity are not well explained by the standard chemical evolution models. Correlations between elemental abundances (e.g. between Ti and $\mathrm{Zn}$ ) could be useful hint to solve these problems.

Large scatter in abundance ratios of neutron-capture elements has been revealed by many studies. Investigation of the detailed distributions of $\mathrm{Sr}$ and $\mathrm{Ba}$ abundance ratios (e.g., exis- 
tence of low-Sr and low-Ba stars) would contain useful information on the origins of these elements.

Some abundance trends (e.g., $\mathrm{Mg} / \mathrm{Fe}$ ratios) could be affected by formation process of the Galactic halo, including accretions of dwarf galaxies. The recent rapid progress in the studies of the connection between abundance ratios and kinematics will shed new light also on understanding of the origins of elements in the Universe.

\section{References}

[1] Heger, A., \& Woosley, S. E., Astrophysical Journal 724, 341. (2010)

[2] Ishigaki, M. N., Tominaga, N., Kobayashi, C., \& Nomoto, K., Astrophysical Journal 857, 46 (2018)

[3] Beers, T. C., Preston, G. W., \& Shectman, S. A., Astronomical Journal 103, 1987. (1992)

[4] Christlieb, N., Green, P. J., Wisotzki, L., \& Reimers, D., Astronomy \& Astrophysics, 375, 366. (2001)

[5] McWilliam, A., Preston, G. W., Sneden, C., \& Searle, L., AJ, 109, 2757. (1995)

[6] Ryan, S. G., Norris, J. E., \& Beers, T. C., ApJ, 471, 254. (1996)

[7] Cayrel, R., Depagne, E., Spite, M., Hill, V., Spite, F., François, P., Plez, B., Beers, T., Primas, F., Andersen, J., Barbuy, B., Bonifacio, P., Molaro, P., \& Nordström, B., A\&A, 416, 1117. (2004)

[8] Cohen, J. G., Christlieb, N., McWilliam, A., Shectman, S., Thompson, I., Wasserburg, G. J., Ivans, I., Dehn, M., Karlsson, T., \& Melendez, J., ApJ, 612, 1107. (2004)

[9] Honda, S., Aoki, W., Kajino, T., Ando, H., Beers, T. C., Izumiura, H., Sadakane, K., \& Takada-Hidai, M., ApJ, 607, 474. (2004)

[10] Yanny, B. et al., AJ, 137, 4377. (2009)

[11] Zhao, G., Zhao, Y.-H., Chu, Y.-Q., Jing, Y.-P., \& Deng, L.-C., Research in Astronomy and Astrophysics, 12, 723. (2012)

[12] Keller, S. C., Schmidt, B. P., Bessell, M. S., Conroy, P. G., Francis, P., Granlund, A., Kowald, E., Oates, A. P., Martin-Jones, T., Preston, T., Tisserand, P., Vaccarella, A., \& Waterson, M. F., PASP, 24, 1. (2007)

[13] Starkenburg, E., Martin, N., Youakim, K. et al., MNRAS, 471, 2587. (2017)

[14] Li, H., Aoki, W., Matsuno, T. et al., submitted to ApJ

[15] Yong, D., Da Costa, G. S., Bessell, M. S., et al., MNRAS, 507, 4102. (2021)

[16] Li, H., Tan, K., \& Zhao, G., ApJS, 238, 16. (2018)

[17] Aoki, W., Li, H., Matsuno, T. et al., submitted to ApJ

[18] Gaia Collaboration et al., A\&A, 616, A1. (2018)

[19] Castelli, F., \& Kurucz, R. L., Modelling of Stellar Atmospheres, 210, A20. (2003)

[20] Romano, D., Karakas, A. I., Tosi, M., \& Matteucci, F., A\&A, 522, A32. (2010)

[21] Kobayashi, C., Karakas, A. I., \& Umeda, H., MNRAS, 414, 3231. (2011)

[22] Prantzos, N., Abia, C., Limongi, M., Chieffi, A., \& Cristallo, S., MNRAS, 476, 3432. (2018)

[23] Nissen, P. E., \& Schuster, W. J., A\&A, 511, L10. (2010)

[24] Helmi, A., ARA\&A, 58, 205. (2020)

[25] Aoki, W. et al., submitted to PASJ 